\title{
Lagoon Hero: de la gamificación en el aula a la preservación de la biodiversidad
}

Edwin Andrés Torres-Parra

Universidad Cooperativa de Colombia

edwincomic@gmail.com

Ismenia Lilibeth Linares-Gómez

Universidad Cooperativa de Colombia

ismenia.linaresgom@campusucc.edu.co

Fency Vianey Martínez-Bejarano

Universidad Cooperativa de Colombia

fencymartinez@hotmail.com

Yiny Paola Cárdenas ${ }^{4}$

Universidad Cooperativa de Colombia

yipacaro22@gmail.com

Jeiner Velandia ${ }^{5}$

Universidad Cooperativa de Colombia

jeiner.velandia@ucc.edu.co

\section{DOI: https://doi.org/10.21158/2357514x.v7.n2.2019.2548}

Cómo citar este artículo: Torres-Parra, E. A.; Linares-Gómez, I. L.; Martínez-Bejarano, F. V.; Cárdenas, Y. P.; Velandia, J. (2019). Lagoon Hero: de la gamificación en el aula a la preservación de la biodiversidad. Revista Virtu@Imente, 7(2), 73-94. DOI: https://doi.org/10.21158/2357514x.v7.n2.2019.2548

Fecha de recepción: 16 de abril 2019

Fecha de aprobación: 01 de junio de 2019
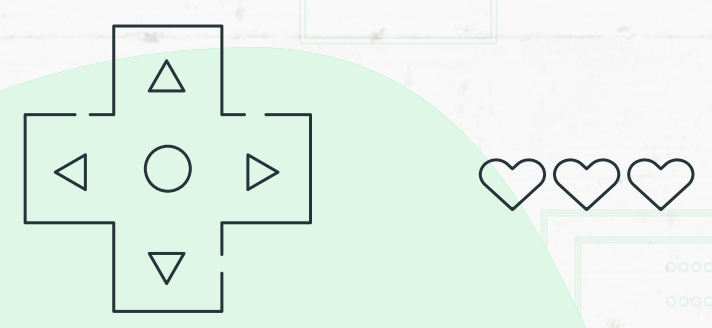

${ }_{1}^{1}$ Magister en Informática Aplicada a la Educación de la Universidad Cooperativa de Colombia, Especialista en Multimedia para la Educación de la Universidad Cooperativa de Colombia, Profesional en Diseño Gráfico, Tecnólogo en Animación 3D. ORCID: https://orcid.org/0000-0002-0348-9425

Licenciada en Educación Básica con énfasis en Humanidades y Lengua Castellana, Especialista en Multimedia para la docencia y Magister en Informática aplicada a la Educación de la Universidad Cooperativa de Colombia. Actualmente Docente del magisterio del Municipio de Soacha. ORCID: https://orcid.org/0000-0003-4789-9984

Licenciada en educación Básica Primaria con Enfasis en Ciencias Naturales de la Universidad del Tolima. Magister en Informática aplicada a la Educación de la Docencia de la Universidad Cooperativa de Colombia. ORCID: https://orcid.org/0000-0001-7393-9746

${ }^{4}$ Licenciada en química de la Universidad Pedagógica Nacional, Especialista en Ambientes Virtuales de Aprendizaje mediante beca de la OEA y el instituto de formación docente Virtual Educa Argentina, Magíster en Docencia de la Química de la Universidad Pedagógica Nacional. ORCID: https://orcid.org/0000-0002-6841-6825

${ }_{5}^{5}$ Magister TI aplicadas a la Educación Diseñador de contenidos educativos, Ambientes Virtuales de Aprendizaje (AVA) y Objetos Virtuales de Aprendizaje (OVA). (Articulate / Captive) Diseñador instruccional con experiencia en el desarrollo de materiales digitales, en el ámbito educativo. ORCID: https://orcid.org/0000-0002-1727-2059 


\section{RESUMEN}

El presente artículo establece la implementación del modelo ADDIE —análisis, diseño, desarrollo, implementación y evaluación- en el diseño de un aplicativo móvil como componente tecnológico de una propuesta didáctica para el fomento de actitudes de cuidado y preservación de la biodiversidad de los humedales de Cundinamarca, Colombia. El aplicativo está orientado metodológicamente bajo los lineamientos de investigación acción, cuyo objetivo es reflexionar y transformar el entorno de las prácticas pedagógicas en el que se ejecuta la investigación; por eso como estrategia para el aprendizaje significativo se utiliza la gamificación y la realidad aumentada, pues se identificaron como tendencias pedagógicas y tecnológicas que en procesos educativos actuales permiten generar escenarios y herramientas para el fomento o el desarrollo de competencias disciplinares de maneras diferentes, vivenciales y acordes con las demandas de la sociedad del conocimiento. Como parte de las conclusiones se identificó que este tipo de herramientas no solo causa efectos positivos en los procesos de aprendizaje de los estudiantes, sino que también son un aporte para los docentes, pues facilitan la apropiación de conceptos de forma atractiva y les muestra cómo la evaluación formativa, mediante la implementación de la actividad interactiva, hace que la evaluación sea menos intrusiva, logrando un mejor desempeño y el cumplimiento de las competencias por parte de los estudiantes.

Palabras clave: prácticas pedagógicas; aprendizaje significativo; gamificación; realidad aumentada; tendencias pedagógicas; tecnología en procesos educativos. 


\section{Lagoon Hero: from gamification in the classroom to the preservation of biodiversity}

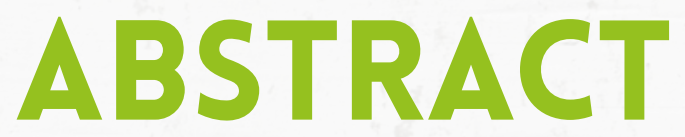

This article establishes the implementation of the ADDIE model -analysis, design, development, implementation, and evaluation- in the design of a mobile application as the technological component of a didactic proposal for the promotion of attitudes of care and preservation of the biodiversity in the wetlands in Cundinamarca, Colombia. The application is methodologically oriented under the action research guidelines, whose objective is to reflect and transform the environment of the pedagogical practices in which the research takes place; therefore, as a strategy for significant learning, we used gamification and augmented reality, since they were identified as pedagogical and technological trends that in current educational processes allow the generation of scenarios and tools for the promotion or development of disciplinary competences in different, experiential ways and according to the demands of the society of knowledge. As part of the conclusions, it was possible to identify that this type of tools not only cause positive effects in the learning processes of students, but it is also a contribution for teachers, since it facilitates the appropriation of concepts in an attractive way and shows them how formative evaluation, by means of the implementation of the interactive activity, makes the evaluation less intrusive, achieving better performance and compliance with competencies on the part of students.

Keywords: pedagogical practices; meaningful learning; gamification; augmented reality; pedagogical trends; technology in educational processes. 


\section{Lagoon Hero: da gamificação em sala de aula à preservação da biodiversidade}

\section{RESUMO}

Este artigo estabelece a implementação do modelo ADDIE -análise, design, desenvolvimento, implementação e avaliação- no design de uma aplicação móvel como componente tecnológico de uma proposta didática para promover atitudes de cuidado e preservação da biodiversidade das áreas úmidas de Cundinamarca, Colômbia. A aplicação é metodologicamente orientada de acordo as diretrizes da pesquisa-ação, cujo objetivo é refletir e transformar o ambiente de práticas pedagógicas em que a pesquisa é realizada; por isso, a gamificação e a realidade aumentada são utilizadas como estratégia de aprendizagem significativa, uma vez que foram identificadas como tendências pedagógicas e tecnológicas que, nos processos educacionais atuais, permitem gerar cenários e ferramentas para a promoção ou desenvolvimento de competências disciplinares em diferentes contextos experimentais de acordo com as demandas da sociedade do conhecimento. Como parte das conclusões, identificouse que esse tipo de ferramenta não apenas causa efeitos positivos nos processos de aprendizagem dos alunos, mas também contribui para os professores, pois facilita a apropriação de conceitos de maneira atraente e mostra como a avaliação formativa, por meio da implementação da atividade interativa, torna a avaliação menos intrusiva, alcançando melhor desempenho e o cumprimento de competências pelos alunos.

Palavras-chave: práticas pedagógicas; aprendizagem significativa; gamificação; realidade aumentada; tendências pedagógicas; tecnologia em processos educacionais. 


\section{Lagoon Hero:du jeux sérieux en classe à la préservation de la biodiversité}

\section{RÉSUMÉ}

Cet article établit la mise en place du modèle ADDIE -analyse, conception, développement, mise en œuvre et évaluation-- pour la conception d'une application mobile comme composant technologique d'une proposition didactique de promotion des comportements de protection et de préservation de la biodiversité des zones humides du département de Cundinamarca, Colombie. La méthodologie de création de cette application s'oriente selon les processus d'investigation-active dont l'objectif est de refléter et transformer l'environnement des pratiques pédagogiques dans lequel est réalisée cette investigation. Voilá pourquoi le jeux sérieux et la réalité augmentée sont utilisés comme stratégie pour un apprentissage augmenté dans la mesure où il s'agit de tendances pédagogiques et technologiques permettant, dans les processus éducatifs actuels, de générer des scénarios et des outils de promotion ou de développement de compétences disciplinaires dans différents domaines d'expérimentation et conformément aux exigences de la société de la connaissance. En conclusion, il est démontré que ces outils ont non seulement des effets positifs sur les processus d'apprentissage mais qu'ils représentent une contribution positive pour les enseignants car ils facilitent l'appropriation des concepts de manière attrayante et rendent l'évaluation formative moins intrusive à travers la mise en œuvre d'activités interactives, améliorant ainsi les performances et l'assimilation des compétences par les étudiants.

Mots-clés: pratiques pédagogiques; apprentissage significatif; jeux sérieux; réalité augmentée; tendances pédagogiques; technologie des processus éducatifs. 


\section{Introducción}

En busca de una construcción de conocimiento con una función social se genera la necesidad de diseñar propuestas didácticas acordes con las demandas de la sociedad del conocimiento, las cuales permitan fomentar actitudes hacia el cuidado y la preservación de la biodiversidad inmersa en ecosistemas, como, por ejemplo, los humedales. El presente artículo da cuenta de la implementación del modelo ADDIE para el diseño un aplicativo móvil acorde a las necesidades de la población a impactar y enfocado en características propias de los humedales de Cundinamarca, Colombia, luego de la identificación de actitudes y comportamientos de estudiantes de básica secundaria con respecto a la interacción, el cuidado y la preservación de la biodiversidad. De acuerdo con lo anterior, se dará a conocer la problemática que demandó la necesidad del diseño del aplicativo, la justificación, los referentes teóricos y el modelo ADDIE para su diseño.

\section{Problemática}

Recuperar y evitar la degradación de las diferentes reservas hídricas que abastecen de bienes y servicios ambientales al país es una tarea que no debe adjudicarse solo a personas encargadas del componente ambiental desde una mirada disciplinar o política, sino también desde el campo educativo y social, a partir de la formación permanente en diferentes momentos y haciendo uso de distintos medios. Según estudios presentados por las diferentes corporaciones autónomas regionales que coordinan el proceso de preparación de los planes, los programas y los proyectos de desarrollo ambiental en materia de protección del medio ambiente y los recursos naturales renovables, de manera que se asegure la armonía y coherencia de las políticas y acciones adoptadas por las distintas entidades territoriales
(Ley 99 de 1993, art. 31), se manifiesta la pérdida de terreno de estos ecosistemas acuáticos por diversas problemáticas naturales y antropogénicas, lo que convierte los humedales en lugares de muerte y desolación, de pérdida de fauna y flora endémica, así como en depósitos de albergue de basuras y escombros, de modo que se altera el equilibrio ecológico y la dinámica natural de estas reservas.

En general, las zonas de alta montaña, subandinas y de tierras bajas, que corresponden a la jurisdicción de la CAR, han sido consideradas como zonas prioritarias para adelantar acciones de conservación en razón a sus características biofísicas (CAR, 2011). Pero, a pesar de los esfuerzos gubernamentales, se encuentra un gran número de 
debilidades: la capacidad de estudios de carga sobre la zona, la insuficiente señalización, el difícil acceso o la falta de un inventario de fauna y flora para los lugareños o visitantes, entre otros. Esta preocupación se centra en estos últimos, quienes por cercanía tienen más acceso a los ambientes naturales, de modo que su falta de conocimiento de las especies, tanto de fauna como de flora, lleva a que las acciones que se desarrollan en el área, bien sean positivas o bien negativas, creen daño en el ambiente natural. Estas manifestaciones enmascaran la trayectoria hacia la inviabilidad del sistema, en tanto que la señal humana se devela únicamente en las etapas tardías de la trayectoria de degradación, cuando ya son pocas las posibilidades de acción (Franco-Vidal, RuizAgudelo, Delgado, Andrade y Guzmán, 2015). Lo anterior refleja cómo la siembra de especies invasoras puede generar un efecto contrario a ayudar el ecosistema y a las especies endémicas, así como mitigar el antrópico que causa la visita a ambientes naturales sin ninguna normatividad o conocimiento del espacio natural.

Si bien entre las acciones municipales se adelantan procesos de alfabetización en torno al cuidado y la protección de los humedales, estas capacitaciones son intermitentes debido a los cambios en las políticas de cada una de las entidades. Por otra parte, la información que se comparte se encuentra en libros impresos o digitalizada en la web, lo cual dificulta el acceso a los inventarios de fauna y flora que crean los entes territoriales.

Otras entidades que apoyan el proceso de alfabetización ambiental son las instituciones educativas, las cuales asumen el compromiso con sus reservas hídricas y se enfocan en desarrollar diferentes herramientas didácticas orientadas a la preservación y el cuidado de la biodiversidad desde un ambiente natural, con la finalidad de crear conciencia y sensibilizar a la comunidad. No obstante, se evidencia que esto ha sido un factor limitante, ya que las herramientas planteadas hasta el momento no emulan la realidad ni la magnitud de la pérdida de estos espacios naturales. Por otra parte, las salidas de campo implican riesgos de difícil acceso, degradación antrópica del ecosistema y diversos costos que varían según el hábitat, todo lo cual impide un acercamiento a la realidad.

De acuerdo con lo anterior, se hace indispensable que la educación y el quehacer docente, desde sus prácticas, tengan un efecto en los estudiantes, como, por ejemplo, tomar conciencia ambiental, por ser ellos parte de una comunidad en la que es necesario reproducir la formación ambiental obtenida y generar así un impacto en la sociedad. Reconociendo que es la educación la que puede dar una solución ante las diferentes problemáticas ambientales (Rengifo-Rengifo, Quitiaquez-Segura y Mora-Córdoba, 2012, p. 22), «la educación ambiental como proceso se centra en fomentar actitudes, destrezas y habilidades necesarias para aprender y apreciar las interrelaciones del ser humano con la naturaleza, que le permita generar conciencia para favorecer el desarrollo sustentable». Para esto se plantea como pregunta orientadora la siguiente: ¿cómo diseñar un aplicativo móvil basado en la gamificación con el fin de fomentar el cuidado y la preservación de la biodiversidad? 


\section{3. ¿Por qué es necesario?}

Los ambientes naturales han sido degradados a causa de las prácticas antrópicas que se generan a gran escala, en especial en los alrededores de los humedales y las reservas hídricas, lo cual disminuye los bienes y los servicios que estos ofrecen a la comunidad. A causa de la desinformación, el desinterés, el uso de políticas no favorables, el crecimiento poblacional, el uso de estos terrenos para la construcción de viviendas de interés social y la falta de sentido de pertenencia por la conservación de estos espacios se produce su deterioro y pronta extinción, causando un desequilibrio en el ecosistema que da lugar a la pérdida de estos ambientes naturales y, en especial, de sus especies endémicas. Así se impide que próximas generaciones gocen de conocer de este tipo de espacios junto con su fauna y flora. Estas acciones se pueden asociar a una falta de conciencia, tal como lo plantea Sandoval (2011) citado en Mosquera y Oese (2015, p. 13):

Hace falta formar una conciencia ambiental dentro del ser humano, misma que se ha perdido con el paso del tiempo, y los avances tecnológicos, ya que cada vez se busca «mejorar» los medios de vida del hombre, quitándole al medio ambiente lo que se pueda, sin cuidarlo, sin tratar de mantener un equilibrio, olvidándonos de que es nuestra casa y si lo destruimos, lentamente nos destruimos a nosotros mismos.
En la sabana de Bogotá se encuentra la mayor cantidad de humedales que comprende la zona andina en Colombia. Su importancia radica en la fauna y flora que allí se encuentra. Según resultados del Global Big Day (CNN, 2018), Colombia ocupa el primer puesto en el avistamiento y la diversidad de aves, gracias a las reservas hídricas con las cuales cuenta el país, puesto que ayudan a la migración y hábitat a la hora de tener crías. También es el nicho para cientos de especies de reptiles y roedores que equilibran la dinámica poblacional, sumado a las especies endémicas de flora, productores primarios y alimento faunístico, reguladores de la calidad del agua, entre otros. Sin embargo, la deforestación, la contaminación y el aumento de nutrientes provenientes de aguas residuales mal tratadas conllevan a la degradación y la pérdida de estos ecosistemas y, por ende, a la extinción de diversas especies, en especial de las migratorias.

Por esta razón es imprescindible que la educación marche a la par del avance de las tecnologías, en las que la implementación de estas debe ser aplicable y útil en la práctica pedagógica, apoyadas en la interdisciplinariedad, bajo la cual se fomenta un aprendizaje significativo y el desarrollo de competencias que le permitan plantear soluciones al problema ambiental que aqueja al contexto. 


\section{Referentes teóricos}

La gamificación es en la actualidad una tendencia educativa a considerar en los procesos de aula, ya que, de acuerdo con Marín y Hierro (2013, p. 13), es,

\begin{abstract}
una técnica, un método y una estrategia a la vez. Parte del conocimiento de los elementos que hacen atractivos a los juegos e identifica, dentro de una actividad, tarea o mensaje determinado, en un entorno de no-juego, aquellos aspectos susceptibles de ser convertidos en juego o dinámicas lúdicas. Todo ello para conseguir una vinculación especial con los usuarios, incentivar un cambio de comportamiento o transmitir un mensaje o contenido. Es decir, crear una experiencia motivadora, que permita al estudiante interiorizar su aprendizaje, implementando estas metodologías permite nutrir el proceso y tomar de manera positiva un interés del usuario, como lo son los juegos.
\end{abstract}

Por ende, la gamificación se considera un medio en el que el estudiante adquiere capacidades para plantear soluciones a diferentes situaciones o resolver problemas mediante su intervención en el juego, promoviendo de esta manera el aprendizaje (Kaap, 2012; Zichermann y Cunningham, 2011).

Por otra parte, la gamificación debe contar con ciertas cualidades que es importante tener en cuenta al momento de aplicarla en el ámbito educativo. Deterding, Dixon, Khaled y Nacke (2011) señalan que el propósito de la gamificación es el «diseño de videojuegos en contextos que no son de juego para hacer que un producto, servicio o aplicación sea más divertido atractivo y motivador». Para
Burke (2011), son «actividades divertidas», mientras que, de acuerdo con Zichermann (2012), buscan «involucrar a los usuarios». En consecuencia, la gamificación es, en esencia, motivación, y la diversión en actividades que involucran el aprendizaje, características no exclusivas de actividades como la gamificación, sino presentes en toda actividad con fines educativos.

Según la experta en diseño de video juegos Jane McGonical (2011), los juegos proporcionan una experiencia divertida porque ofrecen un espacio seguro para fallar y aprender, así como para retroalimentar a los usuarios, además de que permiten la conexión con otros usuarios. Así, se busca que el usuario se apropie del juego y esté en capacidad de intervenir en él, de manera que se enriquezca aún más su experiencia (Kapp, 2012). Esta es una de las mayores razones por la cuales la gamificación combina el juego y la retroalimentación, de modo que permite crear un ambiente mucho más atractivo a la escuela de hoy.

Foncubierta y Rodríguez (2014) destacan la importancia del uso de herramientas tecnológicas en la gamificación y cómo esta combinación potencializa los procesos de aprendizaje porque permite llevar a los estudiantes fuera del aula, aprovechando los espacios en pro del aprendizaje. Para lograr esto, los autores señalan la importancia del diseño objetivo de la actividad gamificada, de manera que esto esté ligado a los elementos, los criterios pedagógicos y los recursos que se van a utilizar; en otras palabras, el trasfondo 
pedagógico y, en su mayoría, el grado de motivación que genere, del cual depende el éxito o el acierto de implementación de este tipo de herramientas (p. 4).

Ahora bien, la conexión de la gamificación con el componente emocional es amplia. De hecho, todo lo que atrapa los sentidos o los implica tiene una relación directa con una experiencia de aprendizaje como algo sentido, vivencial y emocionalmente activo. Lo que carece de emoción no llama nuestra atención.

Otro de los componentes de este aplicativo es la realidad aumentada, una tecnología en la que la realidad se extiende gracias a componentes virtuales y cuyo objetivo es ampliar y mejorar la percepción que tienen las personas de su propio entorno, permitiendo una interactividad por medio de la visualización de información que el usuario no puede percibir con sus sentidos. Para Lara y Villareal (2004), la realidad aumentada consiste en integrar señales de video y audio del mundo real, combinadas con imágenes bidimensionales o tridimensionales generadas por un software desde un computador o diferentes dispositivos. El resultado es una visión dual del mundo real con el mundo virtual, coherentemente estructurado. De esta misma manera, Azuma (1997) lo define como una combinación que vincula información digital con elementos propios de la realidad. Por otra parte, la interacción, entendida como la relación que se crea entre el usuario y el ambiente, en un tiempo real que, por último, permite obtener un registro o visualización en 3D. Estas características hacen posible obtener una definición más clara sobre realidad aumentada en relación con la manera como se aborda en el desarrollo de propuesta.
Por esta razón, la gamificación y la realidad aumentada implementada en el aula requieren de ciertos elementos que hacen de esta experiencia de aprendizaje un anclaje ante los diferentes problemas que se presentan en el interior del aula, como lo son, por ejemplo, la dispersión, la distracción e implicar al estudiante en el proceso de aprendizaje; así mismo, el juego por naturaleza capta la atención, mejora la capacidad de retentiva y memorización, y el provecho de hacer la actividad más vivencial hace que el aprendizaje sea una acción experimental apoyada en la gamificación, para lo cual es indispensable que esta herramienta responda a las necesidades que se presentan en el aula. Así lo describen Foncubierta y Rodríguez (2014, p. 12):

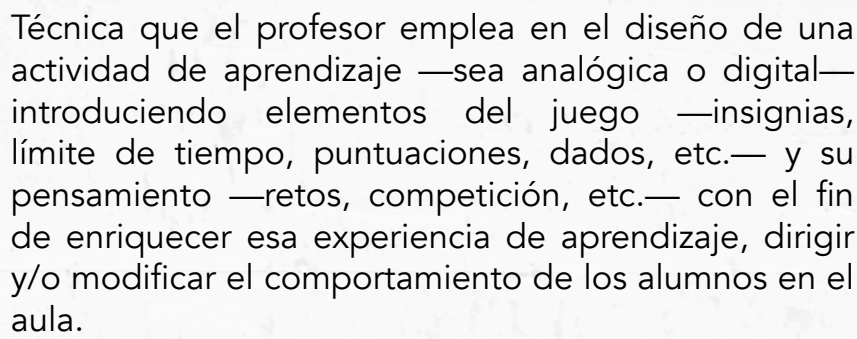
actividad de aprendizaje — sea analógica o digitalintroduciendo elementos del juego -insignias, límite de tiempo, puntuaciones, dados, etc. - y su pensamiento - retos, competición, etc.- con el fin de enriquecer esa experiencia de aprendizaje, dirigir y/o modificar el comportamiento de los alumnos en el aula.

De esta manera, la implementación debe ser dinámica, de modo que permita la experimentación para la construcción del conocimiento, en el cual se buscan los recursos didácticos para promover los valores, las aptitudes, las destrezas y las habilidades útiles que permiten buscar soluciones responsables con el entorno. Esto en consideración a que el constructivismo es un proceso de acción social, es decir, se trata de una interacción en un contexto que también se genera de dicha construcción, lo que permite la participación activa de maestros y estudiantes. Tal como señala Cubero (2005, p. 45): 
La construcción de conocimiento debe tener un carácter social, en un doble sentido: se aprende en la interacción social, y lo que se aprende está determinado socialmente. Las personas realizan una construcción conjunta del conocimiento, negociando los significados y cooperando en dicha construcción.

Según esta postura, la interacción es la construcción entre el conocimiento y la acción, lo cual desarrolla construcciones sociales dirigidas a los contextos de aprendizaje y a participar de manera activa en la gestión del medio ambiente.

Ahora bien, la gamificación y la realidad aumentada se convierten, entonces, en el pilar didáctico para el diseño del aplicativo Lagoon hero, consolidándose como el eje vertebral en cada una de las etapas del modelo instruccional ADDIE —análisis, diseño, desarrollo, implementación y evaluación-, usado para el diseño del aplicativo en conformidad con las necesidades particulares que dieron inicio a la presente investigación, como lo son el fomento de actitudes de cuidado y la preservación de la biodiversidad a partir de un aplicativo tecnológico.
Frente a este tema es posible encontrar diferentes concepciones sobre el modelo del diseño instruccional. Benítez (2010) identifica y caracteriza cuatro generaciones en los modelos de desarrollo instruccional con base en la teoría del aprendizaje en la que se respalda. Frente a la herramienta a implementar, se sustentará bajo el planteamiento de la década de los noventa del siglo XX, la cual se orienta bajo la teoría del constructivismo y se centra en el protagonismo del estudiante en su proceso de aprendizaje. Así mismo, serán importantes también las acciones formativas — de modo que se desarrolla la creatividad y no contenidos específicos por disciplinas - en las que el conocimiento se construye a partir de las experiencias y con la opinión del estudiante y sus saberes previos, de manera que se fomenten metodologías dirigidas al aprendizaje significativo. En la tabla 1 se presenta de manera detallada el abordaje de cada una de las etapas ejecutadas para tal fin. 
Tabla 1. Diseño Instruccional ADDIE de Lagoon hero

\begin{tabular}{|c|c|c|c|}
\hline \multicolumn{4}{|c|}{$\begin{array}{l}\text { Diseño instruccional-modelo ADDIE } \\
\text { Aplicativo móvil Lagoon Hero }\end{array}$} \\
\hline Etapa & Descripción & Actividades & Evaluación \\
\hline
\end{tabular}




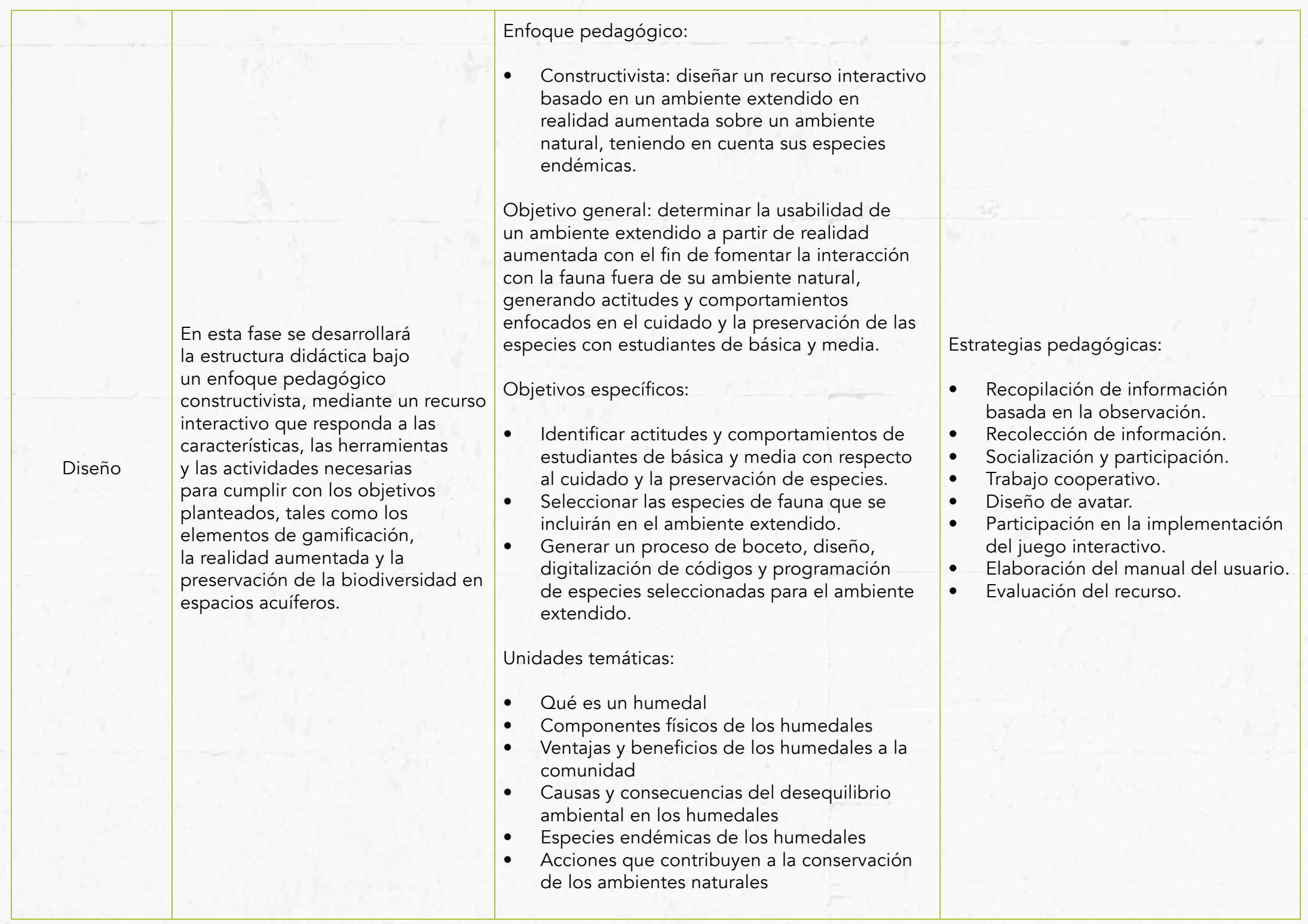




\begin{tabular}{|c|c|c|c|}
\hline & & $\begin{array}{l}\text { Recursos: } \\
\text { - Video sobre cambios que han tenido algunos } \\
\text { humedales. } \\
\text { Dispositivos móviles o tabletas con la } \\
\text { aplicación del juego Lagoon hero. } \\
\text { Actividades: }\end{array}$ & \\
\hline Desarrollo & $\begin{array}{l}\text { En esta fase se elaboran la } \\
\text { planeación de los contenidos y la } \\
\text { evaluación. }\end{array}$ & 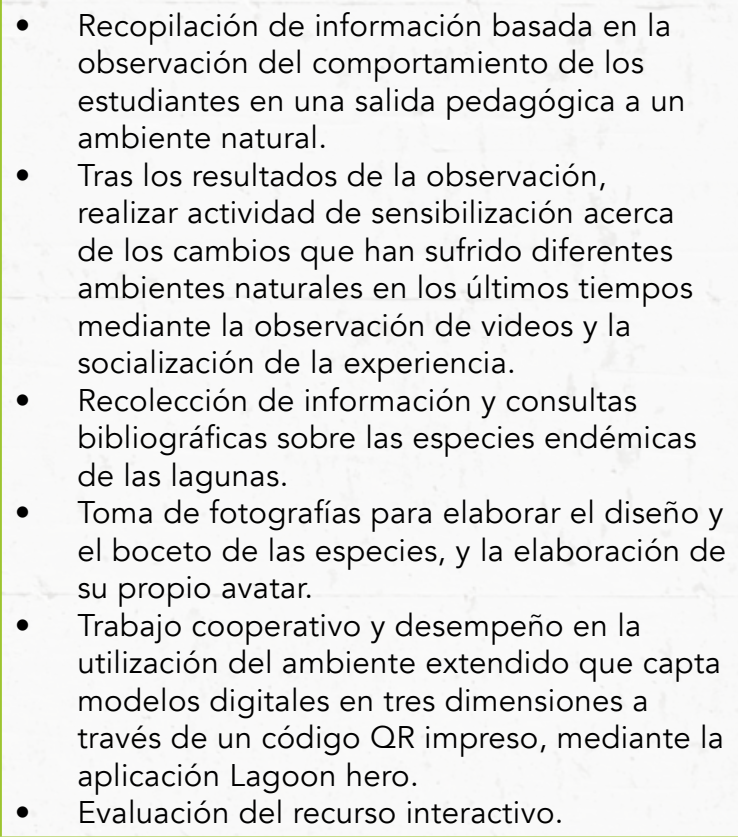 & $\begin{array}{l}\text { Juego interactivo Lagoon hero en cinco } \\
\text { niveles con un grado de complejidad } \\
\text { gradual, acorde con la población y la } \\
\text { temática. } \\
\text { La interacción le permitirá al estudiante } \\
\text { una experiencia creativa, innovadora y } \\
\text { de exploración. }\end{array}$ \\
\hline
\end{tabular}


Edwin Andrés Torres-Parra / Ismenia Lilibeth Linares-Gómez / Fency Vianey Martínez-Bejarano

Yiny Paola Cárdenas / Jeiner Velandia

\begin{tabular}{|c|c|c|c|}
\hline Implementación & $\begin{array}{l}\text { En esta etapa se dará inicio al } \\
\text { proyecto de Lagoon hero, con } \\
\text { estudiantes de educación básica. }\end{array}$ & $\begin{array}{l}\text { - Adaptar los ambientes de aprendizaje, } \\
\text { mediante publicidad, con el fin de dar a } \\
\text { conocer el proyecto. } \\
\text { - } \quad \text { Entrenamiento de docentes y estudiantes. } \\
\text { Pilotaje del proyecto entre los participantes. } \\
\text { Dar a conocer el manual del usuario. } \\
\text { - Puesta en marcha. } \\
\text { Observación de los comportamientos } \\
\text { por parte de los estudiantes durante la } \\
\text { implementación. }\end{array}$ & $\begin{array}{l}\text { Implementación del juego interactivo y } \\
\text { la opinión del usuario. } \\
\text { Resolver los problemas técnicos que se } \\
\text { presenten. }\end{array}$ \\
\hline Evaluación & $\begin{array}{l}\text { En esta fase se desarrolla el } \\
\text { proceso de adecuación del recurso } \\
\text { interactivo; se realizará a lo largo } \\
\text { del proceso del desarrollo a fin } \\
\text { de determinar el alcance de los } \\
\text { objetivos. }\end{array}$ & $\begin{array}{l}\text { Evaluación formativa: } \\
\begin{array}{l}\text { - } \\
\text { Determinar el alcance de los objetivos } \\
\text { - Apocedimentales y actitudinales. } \\
\text { en el juego interactivo. } \\
\text { - Participación, orden y responsabilidad en la } \\
\text { entrega de trabajos asignados. } \\
\text { Evaluación formativa: } \\
\text { - Se realizará una revisión de cada uno de los } \\
\text { niveles que componen el juego interactivo, el } \\
\text { cual se desarrollará a través de una revisión } \\
\text { objetiva. }\end{array}\end{array}$ & $\begin{array}{l}\text { Elaborar una rúbrica para evaluar } \\
\text { el recurso educativo por parte del } \\
\text { usuario. Esto en cuanto a presentación, } \\
\text { elementos del juego y cumplimiento de } \\
\text { objetivos. } \\
\text { Recomendaciones. }\end{array}$ \\
\hline
\end{tabular}

Fuente. Elaboración propia. 
Tabla 2. Vistas de Lagoon Hero

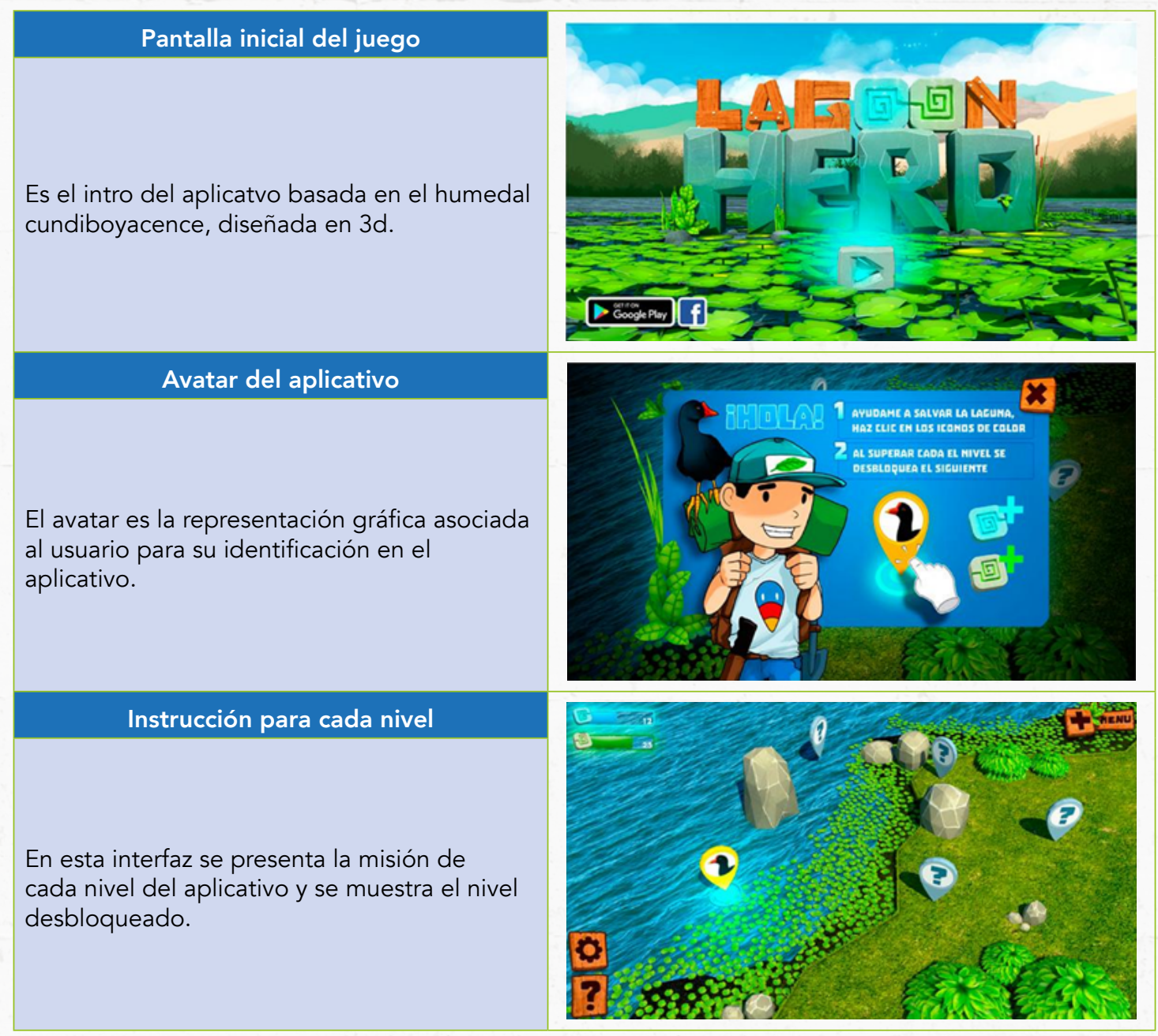




\section{Instruciones de interfaz}

En esta interfaz se explica la función de los elementos de gamificación del aplicativo, tales como: el botón de menú, marcadores de puntaje y botón de ayuda.

\section{Desbloqueo código QR}

Esta interfaz visualiza el nivel superado y habilita el código QR.

\section{Puntajes e instrucciones}

Esta interfaz realiza la retroalimentación e intrucción de cada nivel y los puntajes a obtener.
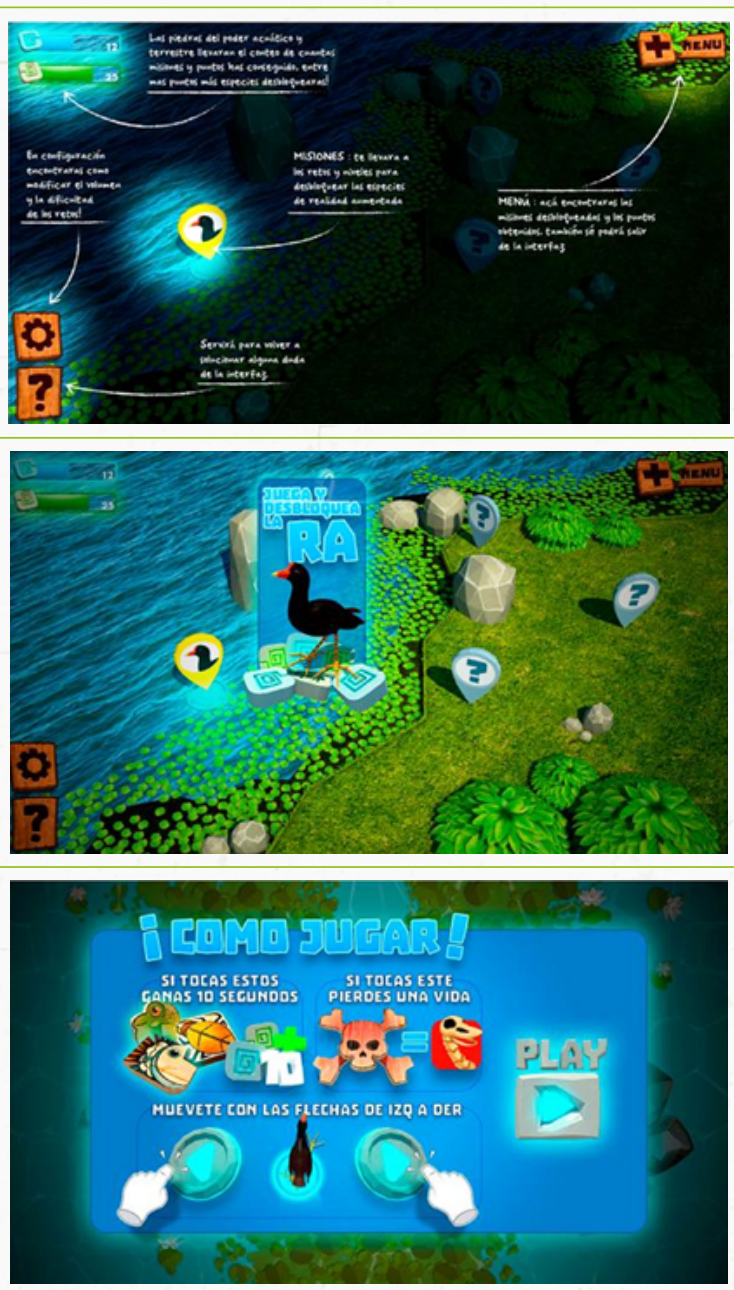
Interfaz primer nivel

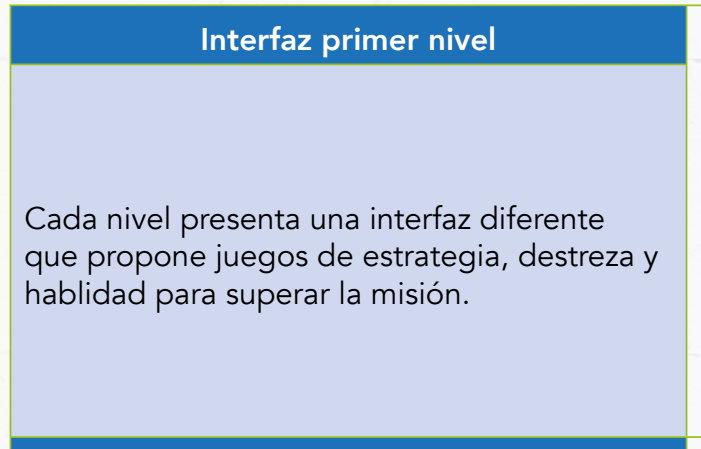

Nivel alcanzado

Esta interfaz al finalizar y superar el nivel

muestra tres botones: reinicio de nivel, inicio de nivel y dirección a la realidad aumentada.

\section{Realidad aumentada}

Al escanear el código QR visualiza la realidad aumentada de la especie desbloqueada.
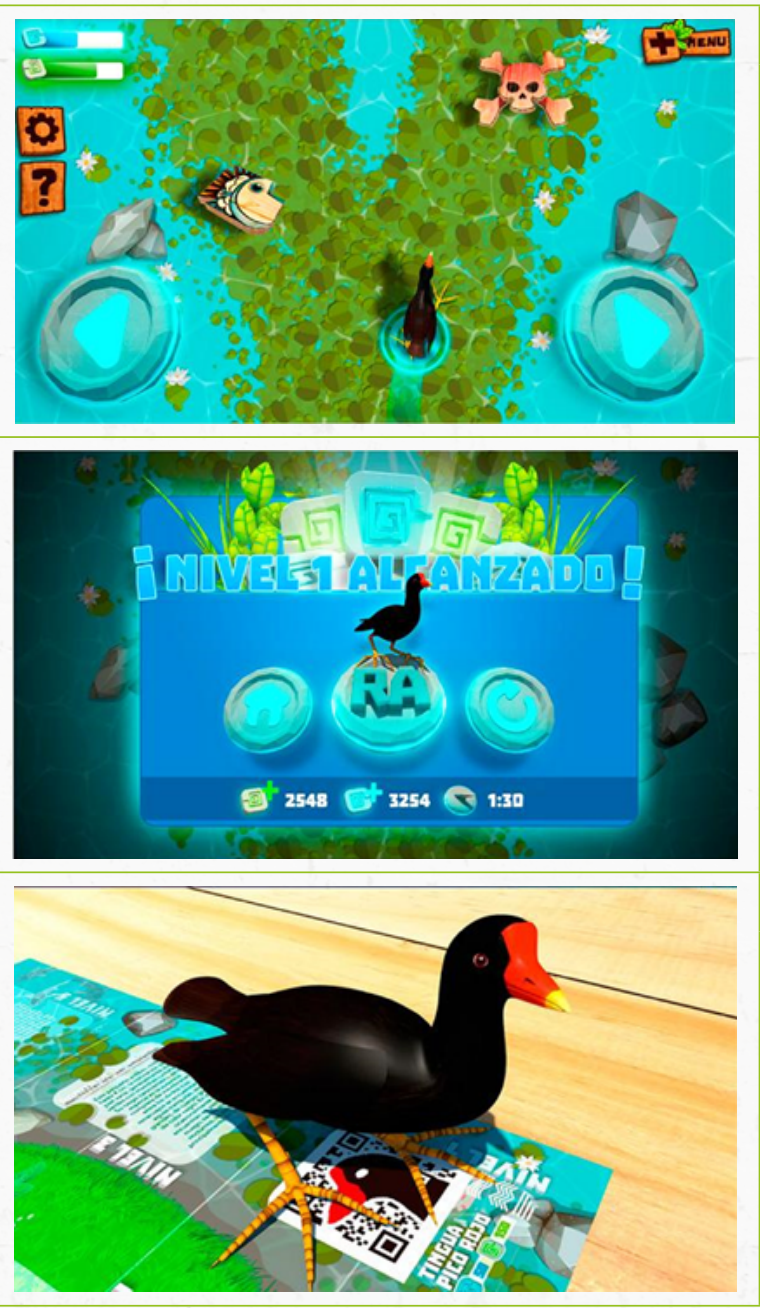

Fuente. Elaboración propia. 


\section{Resultados}

Con Lagoon hero como aplicativo móvil y, a su vez, elemento tecnológico de una propuesta didáctica, se busca fomentar actitudes de cuidado y preservación de la biodiversidad en las instituciones de educación que interactúan con los humedales cundiboyacenses en razón a su cercanía, de modo que los estudiantes se conviertan en guardianes ambientales de contextos reales. Esto se validó en la etapa de implementación y en la evaluación del aplicativo móvil.

Desde esta mirada, el aplicativo móvil, como elemento tecnológico de la propuesta didáctica, está orientado metodológicamente bajo los lineamientos de investigación acción, cuyo objetivo es reflexionar y transformar el entorno de las prácticas pedagógicas en el que se ejecuta la investigación. De esta manera, delimitar esta metodología de la investigación permite su planificación, acción y reflexión, y al ser una construcción en espiral introspectiva esta serie de acciones secuenciales se convierten en la solución del problema (Borroto-Cruz y Aneiros-Riba, 1992, p. 2).

Esta es una forma de indagación introspectiva colectiva, emprendida por participantes en situaciones sociales con el objeto de mejorar la racionalidad y la justicia de sus propias prácticas sociales o educativas, así como su comprensión de esas prácticas y de las situaciones en las que estas tienen lugar.
Una vez expuestas y ejecutadas cada una de las etapas del diseño instruccional ADDIE se logra establecer que la interactividad basada en realidad aumentada y gamificación, en cuanto ambiente inmersivo, puede generar un mayor impacto en los estudiantes con relación a sus actitudes de conservación y al cuidado del medio ambiente. Por tanto, con la integración del aplicativo móvil validado en la propuesta didáctica se busca:

- Aumentar la motivación en la adquisición de ciertas habilidades dentro del contexto de un ambiente inmersivo en el que el estudiante es protagonista.

- Vivir una práctica innovadora, de impacto sensorial, generando curiosidad al resultar atractiva.

- Posibilitar la contradicción o ratificación de un concepto mediante la interactividad, así como materializar el proceso de aprendizaje al relacionar la teoría con la práctica.

- Visualizar el avance de su propio aprendizaje mediante elementos inmersivos. 
Es importante resaltar que el uso este tipo de herramientas no solo causa efectos positivos en los procesos de aprendizaje de los estudiantes, sino que también es un aporte para los docentes, pues facilitan la apropiación de conceptos de forma atractiva y les muestra cómo la evaluación formativa, mediante la implementación de la actividad interactiva, hace que la evaluación sea menos intrusiva, logrando un mejor desempeño y el cumplimiento de las competencias por parte de los estudiantes. A continuación, se describe los aportes a la evaluación desde los elementos de la gamificación.

\section{Conclusiones}

Partir de las necesidades propias de la comunidad cercana a los humedales permite crear recursos educativos focalizados en las problemáticas o en las deficiencias que como ciudadanos debemos solventar frente al cuidado del medio ambiente.

Utilizar las tendencias pedagógicas y tecnológicas en procesos educativos actuales permite generar escenarios y herramientas para el fomento o el desarrollo de competencias disciplinares de maneras diferentes y acordes a las demandas de la sociedad del conocimiento.
El planteamiento de esta herramienta queda abierta a posibles investigaciones. Además, si se tiene en cuenta que este recurso didáctico genera un alto nivel de motivación en los estudiantes, será pertinente medir el nivel de impacto de aplicación con población estudiantil de básica media dentro un contexto rural y urbano. Así, es posible dar continuidad a la investigación planteada, al ser de enfoque exploratorio, puesto que permitirá documentar la experiencia de la implementación del recurso interactivo. 


\section{Referencias}

Azuma, R. (1997). A survey of augmented reality. Presence: Teleoperators and Virtual Environments, 6(4), 355-385. DOI: https://doi.org/10.1162/pres.1997.6.4.355

Borroto-Cruz, R.; Aneiros-Riba, R. (1992). Investigación acción. Escuela Nacional de Salud Pública, Ciudad de la Habana, Cuba. Recuperado de https://bit.ly/2BJszy7

Burke, B. (2011). Innovation insight: gamification adds fun and innovation to inspire engagement. Stamford: Gartner.

Corporación Autónoma Regional de Cundinamrca - CAR. (2011). Humedales del territorio CAR. Bogotá: CAR. Recuperado de https://bit.ly/3eBXTgO

Colombia es Campeona Mundial en avistamiento de aves. (7 de mayo de 2018). CNN. Recuperado de https://cnn.it/2BBb8jw

Cubero-Pérez, R. (2005). Elementos básicos para un constructivismo social. Avances en Psicología Latinoamericana, 23, 4361. Recuperado de https://www.redalyc.org/articulo. oa?id=79902305

Deterding, S.; Dixon, D.; Khaled, R.; Nacke, L. (2011). Gamification: towar a definition. Ponencia presentada en CHI 2011. Vancouver, BC, Canadá, 7-12 de mayo. Recuperado de https://bit.ly/3eypn7l
Foncubierta, J. M.; Rodríguez, C. (2014). Didáctica de la gamificación en la clase de español. Editorial Edinumen. Recuperado de https://bit.ly/31dsspv

Franco-Vidal, L.; Ruiz-Agudelo, C. A.; Delgado, J.; Andrade, G.; Guzmán, A. (2015). Interacciones socioecológicas que perpetúan la degradación de Fúquene, Andes Orientales de Colombia. Ambiente y Desarrollo, 49-66. DOI: https://doi. org/10.11144/Javeriana.ayd19-37.ispd

Kaap, K. (2012). The gamification of learning and instruction. San Francisco: Wiley.

Lara, L. H.; Villareal, J. L. (2004). La realidad aumentada: una tecnología en espera de usuarios. Revista Digital Universitaria UNAM, 5(7), 1-9. Recuperado de https://bit.ly/31eu16w

Ley 99 de 1993. (22 de diciembre de 19993). Por la cual se crea el Ministerio del Medio Ambiente, se reordena el Sector Público encargado de la gestión y conservación del medio ambiente y los recursos naturales renovables, se organiza el Sistema Nacional Ambiental, SINA y se dictan otras disposiciones. Diario Oficial, núm 41.146. Congreso de Colombia

Marín, I.; Hierro, E. (2013). Gamificación: el poder del juego en la gestión empresarial y la conexión con los clientes. Barcelona: Urano-Empresa Activa. 
McGonigal, J. (2011). Reality is broken: why games make us better and how they can change the world. Nueva York: The Penguin Press.

Mosquera, C.; Oese, D. (2015). Conciencia ambiental entre la comunidad educativa caso: institución educativa Diana Oese (Tesis de maestría). Universidad de San Buenaventura, Cali, Colombia. Recuperado de https://bit.ly/2Yv4HY2

Rengifo-Rengifo, B. A.; Quitiaquez-Segura, L.; Mora-Córdoba, F. J. (2012). La educación ambiental una estrategia pedagógica que contribuye a la solución de la problemática ambiental en Colombia. Ponencia presentada en el XII Coloquio Internacional de Geocrítica. Universidad Nacional de Colombia, Bogotá, 7-11 de mayo.

Zichermann, G.; Cunningham, C. (2011). Gamification by design. Implementing game mechanics in web and mobile apps. Canadá: O'Reilly Media. 